\title{
Reversible increase of photocurrents in excimer laser crystallized silicon solar cells
}

\author{
Nilushan K. Mudugamuwa, A. A. D. T. Adikaari, D. M. N. M. Dissanayake V. Stolojan \\ and S. R. P. Silva \\ Advanced Technology Institute, \\ University of Surrey, \\ Guildford, GU2 7XH, UK. \\ n.mudugamuwa@surrey.ac.uk
}

Excimer laser crystallized silicon solar cells fabricated show a steady increment of the current densities with exposure to simulated sunlight, over a 30 minute period. The current density of the amorphous silicon cell under identical conditions remains steady, with no significant change. The process was observed to be reversible upon cooling, and the performance increase is attributed to the energy barrier introduced by the enhanced bandgap of a nanocrystalline silicon middle layer, created as a result of the crystallization. It is suggested that the thermal energy due to prolonged illumination allows carriers to cross the barrier increasing output currents. 
Hydrogenated amorphous silicon (a-Si:H) is one of the most widely used materials for photovoltaics. The pseudo-direct bandgap coupled with the high absorption coefficient, along with reduced costs in deposition compared to crystalline silicon are the main advantages of the material. The high absorption coefficient of a-Si:H also allows one to reduce the thickness of the active layer used. However, light induced degradation of the solar cells, known as the Stabler-Wronski effect, has been a major drawback for the technology.[1] Due to the Stabler-Wronski effect, the devices degrade by $15-30 \%$ from its initial efficiencies.[2] Crystallization of a-Si:H has been studied as one of the potential solutions to this problem.[3,4] Excimer laser crystallization has been a preferred technique for crystallization of a-Si:H deposited on cheap substrates such as glass. Ultraviolet excimer laser energy is highly coupled to a-Si:H and nc-Si:H, due to their high UV absorption coefficients. Upon irradiation, melting and solidifying of the a-Si:H film occur during tens of nanoseconds, with melt depth mainly determined by the laser energy density, often without affecting the underlying substrate. Three major crystallization regimes; partial melting, complete melting and near complete melting, have been reported which primarily depend on energy density and initial film thickness.[5] For photovoltaics, films with adequate thickness for light absorption crystallize in the partial melting regime, resulting in a layered structure.[3] We report the use of the partial melting regime to fabricate solar cells with nanocrystalline silicon (nc$\mathrm{Si}: \mathrm{H}) /$ poly crystalline silicon absorber layers which show increased short circuit current densities $\left(J_{s c} s\right)$ with prolonged solar irradiation, contrary to a-Si:H cells.

Device structures containing indium tin oxide (ITO, $500 \mathrm{~nm}$ )/ chemical vapour deposited $\mathrm{p}^{+}$a-SiC:H $(12 \mathrm{~nm}) / \mathrm{a}-\mathrm{Si}: \mathrm{H}(\sim 400 \mathrm{~nm})$ on glass substrates were obtained from a commercial producer. The samples were irradiated using a Lambda Physik LPX 210i KrF excimer laser operating at $248 \mathrm{~nm}$ with $25 \mathrm{~ns}$ full width half maximum pulse duration. The samples were crystallized in a vacuum chamber with a base pressure of $0.05 \mathrm{~Pa}$. A $4 \times 8 \mathrm{~mm}^{2}$, asymmetric peak profile laser pulse was scanned at $2.5 \mathrm{mms}^{-1}$ with a pulse repetition rate of $50 \mathrm{~Hz}$, for crystallization.[6] The samples were crystallized with 40, 80, 120 and $160 \mathrm{mJcm}^{-2}$ laser energy densities. $60 \mathrm{~nm}$ thick aluminium contacts were evaporated on to the silicon surface after a buffered hydrofluoric acid dip for 20 seconds to remove native oxide layer, resulting in devices with a $5-8 \mathrm{~mm}^{2}$ area.

Crystalline volume fractions of the samples were estimated before evaporation of the metal contacts using Raman spectroscopy with a Renishaw 2000 Ramascope with 782 
$\mathrm{nm}$ excitation.[3] The surface roughness of the devices were also measured using atomic force microscopy with a Digital Instruments Dimension 3100 scanning probe microscope. Figure 1 shows the crystalline volume fractions and the surface roughness of the laser crystallized silicon films and a-Si:H. The crystalline volume fraction of each film increases with the increasing laser energy density, resulting in a maximum of $37 \%$ at $160 \mathrm{mJcm}^{-2}$. The textured transparent electrode used as the light scatterer, ensures that the initial rms roughness of the a-Si:H is $36 \mathrm{~nm}$. The crystallization process increases surface roughness, resulting in a maximum of $47 \mathrm{~nm}$, which is a $30 \%$ increase from the initial roughness. The increased roughness is expected to be favourable rather than detrimental to device performance, enhancing the light scattering capability.

The current-voltage characteristics of the devices were measured under simulated solar irradiation (air mass 1.5,1000 $\mathrm{Wm}^{-2}$ ) using an Oriel 81160 solar simulator.[7] A Keithley 2400 source meter was used for measurements, controlled using custom programmed software. Figure 2 shows the room temperature current density-voltage characteristics. The a-Si:H cell shows a $J_{s c}$ of $7.44 \mathrm{mJcm}^{-2}$, along with an open circuit voltage $\left(V_{o c}\right)$ of 0.6 $\mathrm{V}$, resulting in an efficiency $(\eta)$ of $2.2 \%$ through a fill factor $(F F)$ of $49 \%$. All the devices with laser crystallized silicon absorber layers show inferior performance due to the formation of nc-Si:H.[8] However, further measurements performed on excimer laser crystallized devices after prolonged exposure to simulated sunlight up to 30 minutes in five minute intervals showed an increase in $J_{s c}$. The corresponding temperature under simulated solar irradiation was measured using a thermocouple attached to the aluminium contact using a Fluke $51 \mathrm{~K} / \mathrm{J}$ measurement unit. The temperature was observed to increase with time and saturated at $42^{\circ} \mathrm{C}$ for all the devices after 30 minutes of light exposure. Figure 3 shows the percentage change of $J_{s c}$ of all the devices with time from the room temperature value. The amorphous silicon only device shows no significant increase in $J_{s c}$. The device fabricated at a crystallization energy of $40 \mathrm{mJcm}^{-2}$, show an insignificant increase in $J_{s c}$. This may be attributed to the fact that the device consists of mainly a-Si:H, (96\%, Figure 1), and therefore effectively an amorphous silicon cell. The percentage increase of $J_{s c}$ also increases with crystallization energy density, with a maximum $35 \%$ increase at $160 \mathrm{mJcm}^{-2}$.

Repeat current-voltage measurements on the same devices after cooling (by allowing to cool in atmosphere, in the dark) indicated identical $J_{s c}$ increases, indicating that the process is reversible. This observation suggests that the observed enhancement of 
photocurrents correlates with the other reversible measurement observed due to prolonged light exposure; the cell temperature. However, the thermal energy attained due to the maximum cell temperature measured $\left(42^{\circ} \mathrm{C}\right)$ is insignificant $(\sim 1 \mathrm{meV})$ for a significant increase of carriers in a silicon solar cell. Therefore, in order to investigate the laser crystallized silicon film further, transmission electron microscopy (TEM) was used. Figure 4a shows a cross sectional TEM image of a silicon film excimer laser crystallized at $160 \mathrm{mJcm}^{-2}$, obtained using a Philips CM-200 transmission electron microscope. The crystallizing laser was irradiated from the right hand side of figure 4a, where large grained poly crystalline silicon is formed. The layered structure resulting from partial melting of the initial a-Si:H film is clearly evident, with a nc-Si:H layer sandwiched in the middle. The nc-Si:H is reported to show an enhanced electronic bandgap due to quantum confinement effects from the small silicon nanocrystal structure. [9] The enhancement of the bandgap is reported to be up to $2.3 \mathrm{eV}$, which would be higher compared to unconverted a-Si:H and polycrystalline silicon bandgaps by the sides of the nc-Si:H. [9] The enhanced bandgap would act as a barrier for photo-generated charge carriers travelling under drift conditions upon illumination.[8] Furthermore, it is expected that at the a-Si:H /nc-Si:H and nc-Si:H/poly-Si interfaces, the defect density would be higher, due to grain boundaries. Higher defect states within the bandgap at these interfaces would result in Fermi level pinning, enhancing the potential barrier at these interfaces.[10] Figure 4b shows a band diagram for an excimer laser crystallized silicon cell, showing pinning of the Fermi level at the interfaces, due to grain boundaries. Based on these observations, a model for the reversible increase of photocurrents in excimer laser crystallized silicon solar cells can be proposed. If the defect states at the a-Si:H /nc$\mathrm{Si}: \mathrm{H}$ are quite high, with the abundant carrier generation through photo-excitation, thermalization of carriers with cell temperature increase would effectively relax pinning of Fermi level. Simultaneously, the charges in the conduction band would have their probability of overcoming the barrier at the interface through field emission and thermionic field emission increase. Hence, depending on the temperature of the cell, more charges can be extracted at the contacts, as compared to room temperature operation of the device. This hypothesis is consistent with previous theoretical and experimental reports on charge emission at interface states in silicon. [10,11] The observed temperature dependence of laser crystallized silicon cell may be useful as a 
temperature sensor measuring actual absorber layer temperature of a photovoltaic module.

In conclusion, excimer laser crystallized silicon solar cells were fabricated and increasing $J_{s c}$ were observed with prolonged exposure to AM 1.5 simulated light, contrary to a-Si:H

cell $J_{s c}$ fabricated without the crystallization step. The phenomenon is attributed to the fine-grained nc-Si:H layer formulated during crystallization, sandwiched between an unconverted a-Si:H layer and the polycrystalline silicon layer. The increase of thermal energy due to prolonged light exposure is proposed to increase the probability of photoexcited charges overcoming the interface barrier through field emission and thermionic field emission, aided by the effective relaxation of Fermi level pinning at the interface, resulting in increased $J_{s c}$.

Authors acknowledge EPSRC for a portfolio partnership award which provided funding.

\section{References}

1. D. L. Staebler and C. R. Wronski, Appl. Phys. Lett. 31, 4 (1977).

2. H. Fritzsche, Annu. Rev. Mater. Res. 31, 47 (2001).

3. A. A. D. T. Adikaari and S. R. P. Silva, J. Appl. Phys. 97, 114305 (2005).

4. F. Falk and G. Andra, J. Crys. Grow. 287, 397 (2006).

5. J. S. Im and R. S. Sposili, MRS Bulletin, 21, 39 (1996).

6. A. A. D. T. Adikaari, N. K. Mudugamuwa, S. R. P. Silva, Appl. Phys. Lett. 90, $171912(2007)$

7. International Electrotechnical Commission Code No. IEC 904-1, Geneva, Switzerland, 1987.

8. A. A. D. T. Adikaari, N. K. Mudugamuwa and S. R. P. Silva, unpublished.

9. A. A. D. T Adikaari, J. D. Carey, V. Stolojan, J. L. Keddie, S. R. P. Silva, Nanotechnology, 17, 5412-5416 (2006). 
10. A. W. De Groot and H. C. Card, IEEE Transactions on Electron Devices, 31, 1365 (1984).

11. A. W. De Groot and H. C. Card, IEEE Transactions on Electron Devices, 31, 1370 (1984). 


\section{Figures List}

Figure 1: Crystalline volume fractions and rms roughness of the excimer laser crystallized silicon films with crystallization energy density.

Figure 2: Current density-voltage characteristics of a-Si:H and excimer laser crystallized silicon devices under simulated solar irradiation for each crystallization energy density.

Figure 3: Percentage increase of short circuit current density with crystallization energy densities.

Figure 4: (a) Cross sectional transmission electron micrograph showing the layered silicon structure upon excimer laser crystallization $\left(160 \mathrm{mJcm}^{-2}\right)$. (b) Proposed band diagram for the excimer laser crystallized silicon solar cell showing the energy barrier introduced at interfaces.

Figure 1

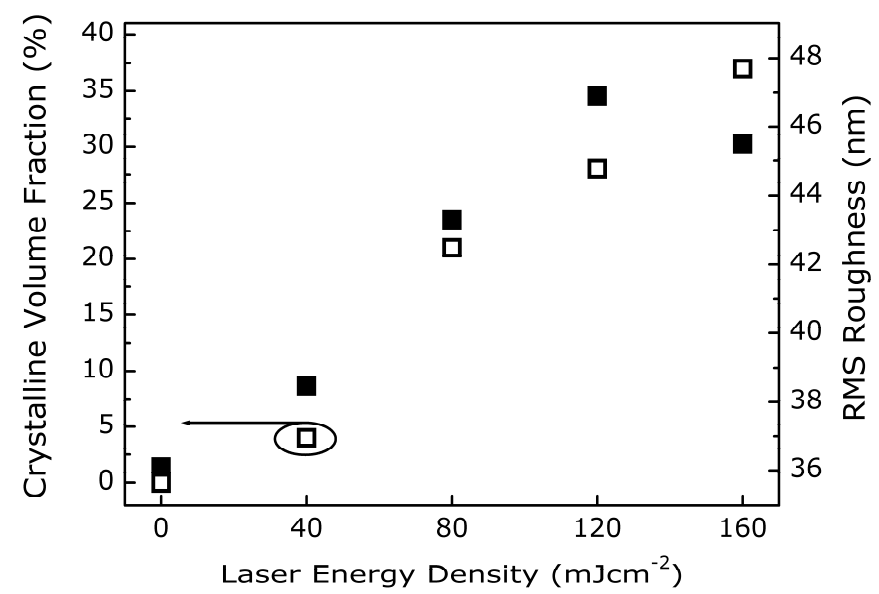


Figure 2

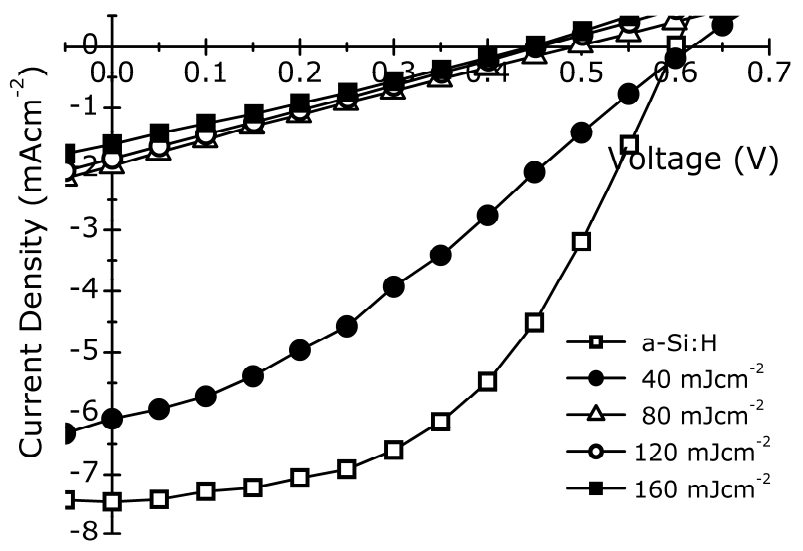

Figure 3

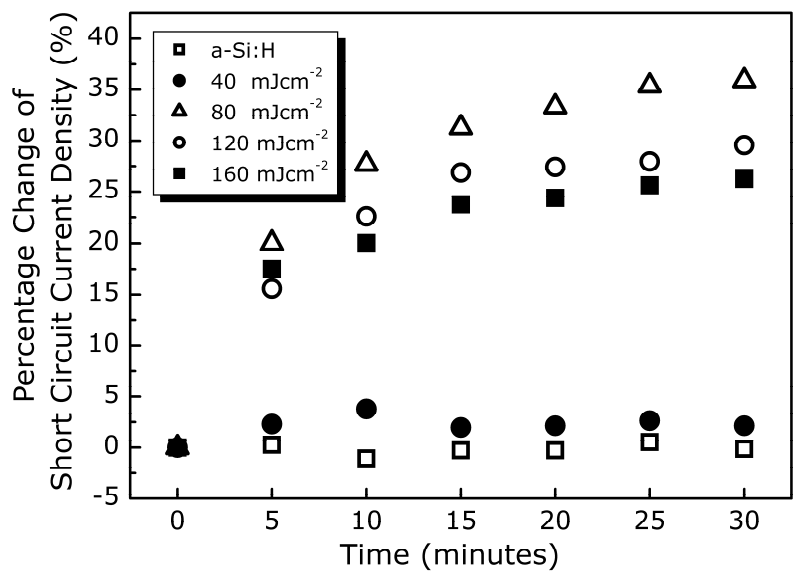

Figure 4
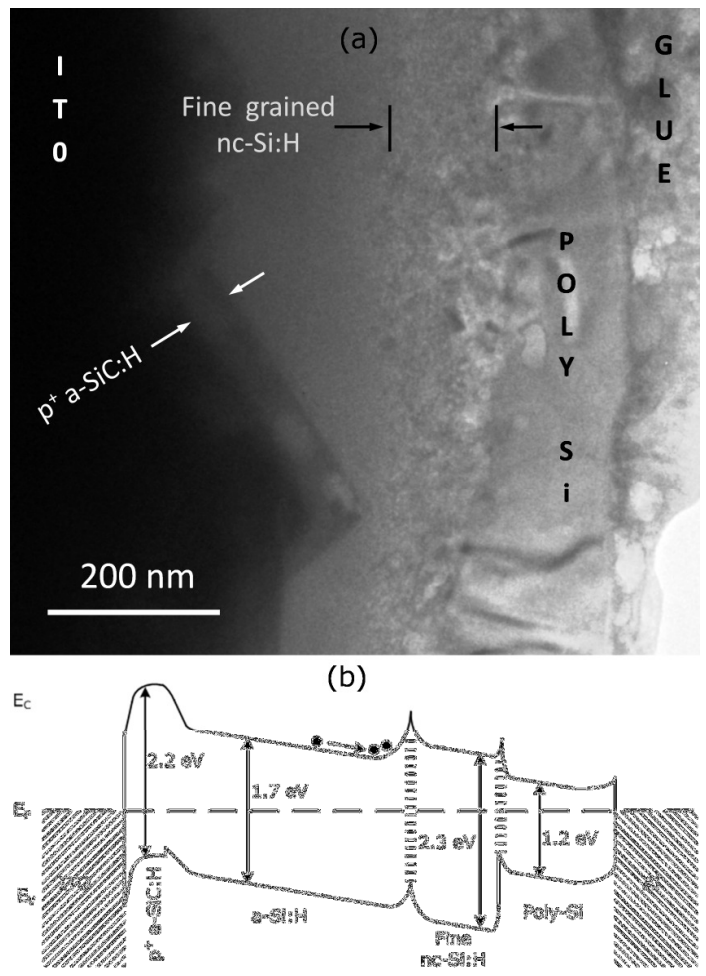